5 Sertório JT, Neto-Neves EM, Dias-Junior CA, et al. Elevated plasma hemoglobin levels increase nitric oxide consumption in experimental and clinical acute pulmonary thromboembolism. Crit Care Med 2013; 41: e118-e124.

6 Levi M. Disseminated intravascular coagulation or extended intravascular coagulation in massive pulmonary embolism. J Thromb Haemost 2010; 8: 1475-1476.

7 Leitner JM, Jilma B, Spiel AO, et al. Massive pulmonary embolism leading to cardiac arrest is associated with consumptive coagulopathy presenting as disseminated intravascular coagulation. J Thromb Haemost 2010; 8: 1477-1482.

8 Watts JA, Gellar MA, Fulkerson MB, et al. Arginase depletes plasma 1-arginine and decreases pulmonary vascular reserve during experimental pulmonary embolism. Pulm Pharmacol Ther 2012; 25: 48-54.

9 Kline JA, Hogg MM, Courtney DM, et al. D-dimer threshold increase with pretest probability unlikely for pulmonary embolism to decrease unnecessary computerized tomographic pulmonary angiography. J Thromb Haemost 2012; 10: 572-581.

10 Voelkel NF, Lobel K, Westcott JY, et al. Nitric oxide-related vasoconstriction in lungs perfused with red cell lysate. FASEB J 1995; 9: 379-386.

11 Nielsen MJ, Moestrup SK. Receptor targeting of hemoglobin mediated by the haptoglobins: roles beyond heme scavenging. Blood 2009; 114: 764-771.

12 Jeffers A, Gladwin MT, Kim-Shapiro DB. Computation of plasma hemoglobin nitric oxide scavenging in hemolytic anemias. Free Radic Biol Med 2006; 41: 1557-1565.

\title{
Prediction of outcome after PEA in chronic thromboembolic pulmonary hypertension using indexed pulmonary artery diameter
}

\author{
To the Editor:
}

Chronic thromboembolic pulmonary hypertension $(\mathrm{CTEPH})$ is associated with considerable morbidity and mortality [1]. It occurs in $2-4 \%$ of patients after acute pulmonary embolism [2]. Pulmonary endarterectomy (PEA) is the treatment of choice to relieve pulmonary artery obstruction in patients with CTEPH and has been remarkably successful [3]. However, in $10-50 \%$ of patients PEA is not possible due to either distal pulmonary vascular obstruction that is surgically inaccessible or significant comorbidities thought to be associated with an unacceptably high risk [4]. Therefore, careful selection of operable candidates is paramount. A correlation between the main pulmonary artery (PA) diameter and pulmonary haemodynamic parameters before PEA have been described in CTEPH patients [5]. We evaluated whether preoperative PA diameter indices could predict the occurrence of mortality and clinical worsening after PEA.

A multidisciplinary panel including pulmonologists, radiologists, cardiologists, and cardiothoracic surgeons reviewed each case. Patients were considered suitable for surgery when they were symptomatic, had an elevated pulmonary vascular resistance (PVR) $\left(>250 \mathrm{dyn} \cdot \mathrm{s} \cdot \mathrm{cm}^{-5}\right)$, segmental or more proximal lesions and no severe comorbidity. Before surgery all patients underwent standardised work-up as described earlier [4]. The study was approved by the local ethical committees of University Hospitals Leuven, Leuven and St Antonius Hospital, Nieuwegein.

According to the study by HeInRICH et al. [5], the widest diameters of the ascending aorta (Ao) and the widest diameter of the main PA perpendicular to its long axis were measured at the level of the bifurcation of the PA. The ratio of the PA and the Ao diameters was calculated [5]. The PA diameter was indexed by body surface area (BSA).

Clinical worsening was defined as the combination of death, need for pulmonary arterial hypertension $(\mathrm{PAH})$ medication initiated after PEA in the presence of persistent or residual pulmonary hypertension (PH) or a 15\% decrease in 6-min walk distance (6MWD) in comparison with the best postoperative value, without improvement in New York Heart Association (NYHA) functional class during follow-up. Clinical worsening was determined by the event which was reached first.

Persistent or residual $\mathrm{PH}$ after PEA was defined as mean pulmonary artery pressure (PAP) $>25 \mathrm{mmHg}$ by right heart catheterisation or systolic PAP $>40 \mathrm{mmHg}$ by echocardiography [4]. 
A preoperative chest computed tomography (CT) scan was available for analysis in 114 of the 161 patients (mean \pm SD age $56.9 \pm 13.8$ years, $57.9 \%$ female) who underwent a PEA in the two centres (61 patients in Nieuwegein and 53 patients in Leuven) between June 2000 and November 2010. 92 (80.7\%) patients had suffered a known pulmonary embolism in the past. The median time between the first episode of pulmonary embolism and the PEA was 2.0 years (range 0.2-51.7 years). 11 (9.6\%) patients had a previous medical history of malignancy. The baseline characteristics are summarised in table 1 . There were no significant differences in baseline characteristics between patients with or without available preoperative CT scan for analysis.

Seven $(6.1 \%)$ patients died within 30 days after PEA, due to right ventricular failure in two patients, thoracic bleeding in two patients, reperfusion oedema in two patients and multi-organ failure in one patient. By multivariate analysis the indexed PA diameter and mean PAP were the only independent preoperative predictors of 30-day mortality after PEA (OR 1.35, 95\% CI 1.01-1.81, p=0.04; and OR 1.21, $95 \%$ CI $1.03-1.42, \mathrm{p}=0.02$, respectively). The immediate postoperative mean PAP in patients who died within 30 days of PEA was $45.6 \pm 11.9 \mathrm{mmHg}$, versus $30.2 \pm 11.6 \mathrm{mmHg}$ in patients who survived, and was a predictor for the occurrence of 30-day mortality (OR 1.11, 95\% CI 1.02-1.20; $\mathrm{p}=0.01$ ). The postoperative PVR in nonsurvivors was $764 \pm 202 \mathrm{dyn} \cdot \mathrm{s} \cdot \mathrm{cm}^{-5}$ versus $367 \pm 211 \mathrm{dyn} \cdot \mathrm{s} \cdot \mathrm{cm}^{-5}$ in survivors, and was also a predictor for mortality within 30 days after surgery (OR 2.11, 95\% CI 1.23-3.61; p=0.007).

During a mean follow-up of 3.2 years (range $0.01-7.8$ years), eight (7.5\%) out of 107 patients who survived surgery died. Only one patient died as the consequence of progression of $\mathrm{PH}$. The other patients died because of sepsis, sudden death, leukaemia, stroke and an overdose, respectively. In two patients the cause of death was unknown. Clinical worsening occurred in $24(22.4 \%)$ out of 107 patients. History of malignancy was present in one $(4.2 \%)$ of these 24 patients. In four out of these 24 patients clinical worsening occurred as the result of death, in 11 patients as a consequence of initiation of PAH specific medication and in nine patients due to the combination of a $15 \%$ decrease in 6MWD without improvement in NYHA functional class.

TABLE 1 Baseline characteristics and predictors for clinical worsening during follow-up after pulmonary endarterectomy using Cox proportional hazards

\begin{tabular}{|c|c|c|c|c|c|}
\hline Variables & Baseline & Univariate HR $(95 \% \mathrm{Cl})$ & p-value & Multivariate HR $(95 \% \mathrm{Cl})$ & p-value \\
\hline Subjects & 114 & 107 & & & \\
\hline Age years & $56.9 \pm 13.8$ & $1.05(1.01-1.09)$ & 0.008 & $1.06(1.01-1.11)$ & 0.02 \\
\hline BSA $\mathrm{m}^{2}$ & $1.9 \pm 0.2$ & $0.98(0.96-1.00)$ & $0.02^{\#}$ & & \\
\hline Systolic BP mmHg & $128.3 \pm 19.8$ & $1.00(0.98-1.02)$ & 0.98 & & \\
\hline Diastolic BP $\mathrm{mmHg}$ & $81.6 \pm 12.7$ & $0.99(0.96-1.02)$ & 0.45 & & \\
\hline \multicolumn{6}{|l|}{ Cardiac evaluation } \\
\hline Mean PAP mmHg & $41.2 \pm 10.2$ & $1.01(0.97-1.05)$ & 0.68 & & \\
\hline PVR dyn $\cdot \mathrm{s} \cdot \mathrm{cm}^{-5}$ & $759 \pm 422$ & $1.09(0.98-1.21)$ & 0.10 & & \\
\hline Cardiac output $\mathrm{L} \cdot \mathrm{min}^{-1}$ & $4.1 \pm 1.2$ & $0.60(0.38-0.95)$ & 0.03 & $0.85(0.52-1.39)$ & 0.52 \\
\hline Heart rate beats per min & $77.1 \pm 14.9$ & $0.98(0.95-1.01)$ & 0.18 & & \\
\hline LVEF \% & $62.2 \pm 9.4$ & $1.05(1.01-1.09)$ & 0.02 & $1.05(1.01-1.10)$ & 0.03 \\
\hline PA diameter $\mathrm{mm}$ & $35.7 \pm 6.0$ & $1.09(1.02-1.16)$ & 0.02 & & \\
\hline Ao diameter $\mathrm{mm}$ & $32.0 \pm 4.7$ & $1.09(1.00-1.18)$ & 0.06 & & \\
\hline PA/Ao & $1.1 \pm 0.2$ & $1.06(0.10-11.4)$ & 0.96 & & \\
\hline PA/BSA $\mathrm{mm} \cdot \mathrm{m}^{-2}$ & $19.0 \pm 3.9$ & $1.29(1.14-1.46)$ & $<0.001$ & $1.20(1.04-1.39)$ & 0.01 \\
\hline
\end{tabular}

Data are presented as $n, n(\%)$ or mean $\pm S D$, unless otherwise stated. HR: hazard ratio; BSA: body surface area; BP: blood pressure; NYHA FC: New York Heart Association functional class; 6MWD: 6-min walk distance; PAP: pulmonary artery pressure; PVR: pulmonary vascular resistance; LVEF: left ventricular ejection fraction; VC: vital capacity; FEV1: forced expiratory volume in $1 \mathrm{~s} ; \mathrm{CT}$ : computed tomography; PA: pulmonary artery; Ao: aorta; " : per $0.1 \mathrm{~m}^{2}$. 
Indexed PA diameter (HR 1.20, 95\% CI 1.04-1.39; $\mathrm{p}=0.01$ ), age (HR 1.06, 95\% CI 1.01-1.11; $\mathrm{p}=0.02)$ and LVEF (HR 1.05, 95\% CI 1.01-1.10; $\mathrm{p}=0.03$ ) were independent preoperative predictors for the occurrence of clinical worsening during follow-up (table 1).

The immediate postoperative mean PAP $(37.3 \pm 12.0 \mathrm{mmHg}$ versus $28.0 \pm 10.6 \mathrm{mmHg}$; HR $1.07,95 \%$ CI $1.03-1.10, \mathrm{p}<0.001)$ and the postoperative PVR $\left(452 \pm 231 \mathrm{dyn} \cdot \mathrm{s} \cdot \mathrm{cm}^{-5}\right.$ versus $323 \pm 188 \mathrm{dyn} \cdot \mathrm{s} \cdot \mathrm{cm}^{-5} ; \mathrm{HR}$ $1.29,95 \%$ CI $1.05-1.59, \mathrm{p}=0.01$ ) were predictors for the occurrence of clinical worsening.

The present study demonstrates that the PA diameter indexed for BSA, is an independent predictor for the occurrence of mortality within 30 days after PEA and for the occurrence of clinical worsening during follow-up in patients with operable CTEPH.

The 30-day mortality rate in our study was $6.1 \%$, which is in agreement with earlier studies [6]. Known preoperative predictors for perioperative mortality are a preoperative increased PAP, ethnicity, preoperative PVR, and 6MWD [3,7]. Interestingly, in our study, the mean PAP and indexed PA diameter at baseline were the only independent preoperative predictors for 30-day mortality after PEA.

Recently, clinical worsening has been used as a composite end-point in PAH trials, as described by MCLAUghlin et al. [8]. During follow-up, clinical worsening occurred in $22 \%$ of patients, mainly driven by the initiation of PAH specific therapy. In search for preoperative predictors for clinical worsening, we identified age, left ventricular ejection fraction and the indexed PA diameter as independent predictors for the occurrence of clinical worsening.

The dilation of the main PA is a common finding in patients with PH. Several studies identified an increased main PA diameter as a reliable predictor of PAH, especially when the ratio of the main PA and Ao diameter is used $[9,10]$.

The association between PA diameter indices and outcome after PEA might be explained by the presence of more extensive disease in patients with higher PA diameters. The dilation of the PA reflects both the degree and the duration of the presence of increased PAP. The reduced compliance of the PA is an important factor in the relationship between the PA diameter and the increased PAP.

Some limitations of our study have to be mentioned. First, it is a retrospective study which might result in lack of data. In 47 patients there was no CT scan available for retrospective analysis because these were performed in a referring hospital. Furthermore, the study was conducted in two tertiary referral centres; therefore, selection bias might be present.

In conclusion, the noninvasively measured PA diameter indices might be a valuable addition in predicting the outcome prior to PEA. However, every patient with CTEPH should be referred to centres with excellence in the management of CTEPH and PEA surgery regardless of prognostic indices.

○ @ERSpublications

Pulmonary artery diameter indices may be a valuable addition in predicting the outcome prior to pulmonary endarterectomy http://ow.ly/qOnYQ

Bastiaan E. Schölzel ${ }^{1}$, Martijn C. Post ${ }^{1,2}$, Steven Dymarkowski ${ }^{3}$, Wim Wuyts ${ }^{4}$, Bart Meyns ${ }^{5}$, Werner Budts ${ }^{2}$, Wim Morshuis ${ }^{6}$, Repke J. Snijder ${ }^{7}$ and Marion Delcroix ${ }^{4}$

${ }^{1}$ Dept of Cardiology, St Antonius Hospital, Nieuwegein, ${ }^{6}$ Dept of Cardio-Thoracic Surgery, St Antonius Hospital, Nieuwegein, and ${ }^{7}$ Dept of Pulmonology, St Antonius Hospital, Nieuwegein, The Netherlands. ${ }^{2}$ Dept of Cardiology, University Hospitals Leuven, Leuven, ${ }^{3}$ Dept of Radiology, University Hospitals Leuven, Leuven, ${ }^{4}$ Dept of Pulmonology, University Hospitals Leuven, Leuven, and ${ }^{5}$ Dept of Cardio-Thoracic Surgery, University Hospitals Leuven, Belgium.

Correspondence: M.C. Post, St Antonius Hospital, Dept of Cardiology, Koekoekslaan 1, 3435 CM Nieuwegein, The Netherlands. E-mail: m.post@antoniusziekenhuis.nl

Received: Oct 062013 | Accepted: Nov 032013

Conflict of interest: Disclosures can be found alongside the online version of this article at www.erj.ersjournals.com

\section{References}

1 Schölzel B, Snijder R, Morshuis W, et al. Clinical worsening after pulmonary endarterectomy in chronic thromboembolic pulmonary hypertension. Neth Heart J 2011; 19: 498-503.

2 Pengo V, Lensing AW, Prins $\mathrm{MH}$, et al. Incidence of chronic thromboembolic pulmonary hypertension after pulmonary embolism. N Engl J Med 2004; 350: 2257-2264.

3 Madani MM, Auger WR, Pretorius V, et al. Pulmonary endarterectomy: recent changes in a single institution's experience of more than 2,700 patients. Ann Thorac Surg 2012; 94: 97-103.

4 Pepke-Zaba J, Delcroix M, Lang I, et al. Chronic thromboembolic pulmonary hypertension (CTEPH): results from an international prospective registry. Circulation 2011; 124: 1973-1981. 
5 Heinrich $\mathrm{M}$, Uder $\mathrm{M}$, Tscholl $\mathrm{D}$, et al. CT scan findings in chronic thromboembolic pulmonary hypertension: predictors of hemodynamic improvement after pulmonary thromboendarterectomy. Chest 2005; 127: 1606-1613. Mayer E, Jenkins D, Lindner J, et al. Surgical management and outcome of patients with chronic thromboembolic pulmonary hypertension: results from an international prospective registry. J Thorac Cardiovasc Surg 2011; 141: 702-710.

7 Condliffe R, Kiely DG, Gibbs JS, et al. Prognostic and aetiological factors in chronic thromboembolic pulmonary hypertension. Eur Respir J 2009; 33: 332-338.

8 McLaughlin VV, Badesch DB, Delcroix M, et al. End points and clinical trial design in pulmonary arterial hypertension. J Am Coll Cardiol 2009; 54: Suppl. 1, S97-S107.

9 Ng CS, Wells AU, Padley SP. A CT sign of chronic pulmonary arterial hypertension: the ratio of main pulmonary artery to aortic diameter. $J$ Thorac Imaging 1999; 14: 270-278.

10 Haimovici JB, Trotman-Dickenson B, Halpern EF, et al. Relationship between pulmonary artery diameter at computed tomography and pulmonary artery pressures at right-sided heart catheterization. Massachusetts General Hospital Lung Transplantation Program. Acad Radiol 1997; 4: 327-334.

\title{
Plasma interleukin-6 adds prognostic information in pulmonary arterial hypertension
}

\author{
To the Editor:
}

Noninvasive biomarkers are needed to aid in making challenging clinical decisions in pulmonary arterial hypertension (PAH). Several biomarkers have been described, but only the natriuretic peptides have gained clinical utility. A key question to answer is whether or not a new biomarker adds independent incremental information [1]. Novel PAH markers may be discovered from new pathobiological pathways, such as inflammation. In particular, interleukin (IL)-6 has been linked with the development of severe pulmonary hypertension in animal models, mimicking the pathology of human disease [2]. IL-6 is a major regulator of the production of C-reactive protein (CRP), a marker of cardiovascular risk [3]. We conducted this study to determine if these inflammatory biomarkers add incremental prognostic information in PAH.

This is a cohort study based on a prospective Biobank. We enrolled patients with idiopathic, heritable, connective tissue-associated and congenital heart disease-associated PAH, as defined by current guidelines [4], between March 2005 and June 2011. The study was approved by the Cleveland Clinic Institutional Review Board. We used ELISA (R\&D Systems Inc., Minneapolis, MN, USA) to measure IL-6, the Abbott platform for highly-sensitive CRP (hsCRP) (Abbott Laboratories, Abbott Park, IL, USA) and a chemiluminescence immunoassay to measure B-type natriuretic peptide (BNP) (ADVIA Centaur XP; Siemens Healthcare Diagnostics, Inc., Tarrytown, NY, USA) Peripheral plasma samples were kept at $-80^{\circ} \mathrm{C}$ until retrieved for the measurement of the biomarkers (September 2011 for hsCRP and BNP, and February 2012 for IL-6). Investigators who performed the biomarker determination were blinded to the study participants' outcomes.

All-cause mortality since the date of study blood sampling was ascertained via manual and automated query of electronic medical records, as well as query of the Social Security Death Index. Lung transplantation was censored. The following data were collected on the same day as blood sampling: New York Heart Association (NYHA) functional classification, 6-min walking distance (6MWD), echocardiography, and per cent predicted diffusing capacity of the lung for carbon monoxide (DLCO \% pred). Right heart catheterisation data obtained closest to the date of study blood sampling were recorded (median time 27 days, interquartile range 5-66 days). Analyses were performed using JMP Pro 9.0.0 (SAS Institute Inc., Cary, NC, USA) and R version 2.14.2 (The R Project for Statistical Computing, http://www.r-project.org/). We used receiver operating characteristic (ROC) curve analysis, Kaplan-Meier curves and Cox models to determine the associations between biomarkers and mortality. We also used recursive partitioning treebased analysis, using the R function "rpart", to identify the group of biomarkers and baseline variables that had optimal discriminatory ability with regards to all-cause mortality. Each possible threshold of biomarker and baseline variable was evaluated to find the split that formed two groups with the greatest difference in 